\title{
Spiraling Bubbles: How Acoustic and Hydrodynamic Forces Compete
}

\author{
Judith Rensen, ${ }^{1}$ Dennis Bosman, ${ }^{1}$ Jacques Magnaudet, ${ }^{2}$ Claus-Dieter Ohl,${ }^{1}$ Andrea Prosperetti, ${ }^{1,3}$ Rüdiger Tögel, ${ }^{1}$ \\ Michel Versluis, ${ }^{1}$ and Detlef Lohse ${ }^{1, *}$ \\ ${ }^{1}$ Department of Applied Physics, University of Twente, 7500 AE Enschede, The Netherlands \\ ${ }^{2}$ Institut de Mécanique des Fluides de Toulouse, 2, Avenue Camille Soula, 31400 Toulouse, France \\ ${ }^{3}$ Department of Mechanical Engineering, The Johns-Hopkins University, Baltimore, Maryland 21218
}

(Received 28 February 2001)

\begin{abstract}
Experiments to study the effect of acoustic forces on individual bubbles in shear flows have been carried out. In the system that we have used, the competition between acoustic and fluid dynamical forces results in a spiraling bubble trajectory. This dynamics is modeled by expressing the balance between Bjerknes and hydrodynamic forces in terms of an ordinary differential equation model, to which a separation of time scales is applied. The success of this model shows that the simple force-balance approach is still meaningful when bubbles are subjected to sound fields.
\end{abstract}

PACS numbers: $43.25 .+\mathrm{y}, 47.55 . \mathrm{Dz}$

Introduction. - The dynamics of a bubble in a flow is determined by buoyancy and hydrodynamic forces. If an acoustic field is also present, the dynamics is modifiedhydrodynamic and acoustic forces now compete. Understanding this competition opens the possibility of controlling the motion of bubbles subjected to a flow by means of an external sound field. Potentially, this possibility of controlling bubbles has numerous applications, such as drug delivery in living systems [1]. A study of the competition between the forces can also give information on fluid dynamical quantities such as the lift and the drag coefficients.

Bubble control through acoustic forces in a stagnant liquid has already been successfully achieved, e.g., in single bubble sonoluminescence [2]. In these experiments, the acoustic Bjerknes force balances the hydrostatic buoyancy force acting on a bubble. To make an extension to situations involving a mean fluid motion, we have performed experiments on a bubble in a quasi-Poiseuille flow in the presence of a standing acoustic wave. An ordinary differential equation (ODE) approach, based on a balance between hydrodynamic and Bjerknes forces, and a separation of the fast acoustic time scale, can well account for the observations. In particular, the model describes the observed spiraling trajectory within the experimental accuracy.

Experiments. - Figure 1 depicts the experimental setup. The test section is a 1-mm-thick glass diffuser embedded in a spherical acoustic glass resonator with a diameter of $80 \mathrm{~mm}$ (1). The cylindrically symmetric diffuser, which penetrates the spherical resonator, is a $60-\mathrm{mm}$-long truncated cone expanding downward from a diameter of $20 \mathrm{~mm}$ to one of $25 \mathrm{~mm}$. The resonator is driven at its main resonance frequency $f_{d} \simeq 20 \mathrm{kHz}$ by two piezo transducers (2), excited by a high-voltage sinusoidal signal delivered by a function generator, amplifier, and impedance matching circuit. The standing spherical wave generated has a pressure antinode at the center of the resonator, and a node at the wall. Except for the diffuser, the components of the hydraulic circuit are made of PVC tubing. The setup is filled with deionized water. The flow is directed downward in the test section and the flow rate is measured with a rotameter (5). The Reynolds number of the flow based on the upper radius of the diffuser can be varied between 100 and 1000 . Bubbles are injected by a bubble generator (3), and are discharged in a large vessel (6).

The bubble motion is recorded from two orthogonal views with a single video camera at 25 frames per second. Later the individual images are transferred and digitally processed onto a computer to extract the bubble trajectory. This includes the steps of edge detection, contour tracking, and calculating the centroid of the bubble in both views. As the bubble motion is restricted inside the narrow diffuser at the center of the resonator, the distortion due to the spherical surface is small. This has been checked with a calibration grid which is also used to measure the effective magnification of the optical setup.

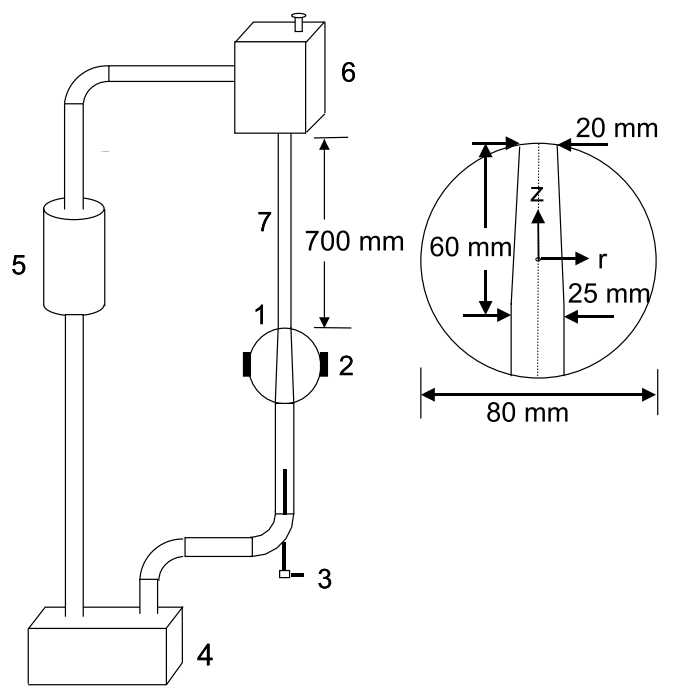

FIG. 1. Experimental setup: (1) test section; (2) piezo elements; (3) bubble generator; (4) pump; (5) flowmeter; (6) vessel with free surface; (7) inlet to the test section. 
In the experiments two different types of bubble trajectories were found: a bubble reaches an equilibrium position and stays there. When the sound field is switched off, the bubble moves out of the test section. However, in case of low driving pressures $(<10 \mathrm{kPa})$, bubbles start to spiral in a vertical plane. In this Letter we focus on this second type of behavior.

To present the data we use a cylindrical coordinate system $(r, z, \theta)$; see Fig. 1 . Oscillations of both the $z$ and $r$ coordinates of the bubble center were observed in the experiments, with hardly any $\theta$ dependence [3]; a typical result is shown in Figs. 2 and 3. In this particular experiment the parameters have the following values: flow rate $Q \approx 10^{-5} \mathrm{~m}^{3} / \mathrm{s}$, equilibrium bubble radius $R_{B 0} \approx 200 \mu \mathrm{m}$, amplitude of the driving pressure $P_{a} \approx 4 \times 10^{3} \mathrm{~Pa}$. The values of $P_{a}, R_{B 0}$, and $Q$ are known only approximately. For this data set the spiraling frequency is $f \approx 0.37 \mathrm{~Hz}$ and the damping constant is $\gamma \approx 0.01 \mathrm{~s}^{-1}$.

Force balance model. - Since the vertical tube upstream of the test section ( 7 in Fig. 1) has a length of 35 bubble diameters, we approximate the flow as fully developed. Furthermore, given the small divergence angle of the diffuser (less than $5^{\circ}$ ), we assume a parabolic velocity distribution given by

$$
U(r, z) \simeq \frac{-2|Q|}{\pi R_{\mathrm{diff}}^{2}(z)}\left(1-\frac{r^{2}}{R_{\mathrm{diff}}^{2}(z)}\right),
$$

where $R_{\text {diff }}(z)$ is the local radius of the diffuser.

To model the bubble behavior, we balance the acoustic (or primary Bjerknes) force $\mathbf{F}_{\mathrm{BJ}}$ against the hydrodynamic forces - inertia $\mathbf{F}_{I}$, added mass $\mathbf{F}_{A}$, lift $\mathbf{F}_{L}$, drag $\mathbf{F}_{D}$, and buoyancy $\mathbf{F}_{G}$. The time scale $f_{d}^{-1}=50 \mu$ s of the volume oscillations of the bubble is much smaller than the time scale of the spiraling motion. Therefore we can carry out a separation of time scales by averaging over one acoustic cycle. In this way, the force balance results in an ODE for the bubble position $\mathbf{x}_{B}$ :

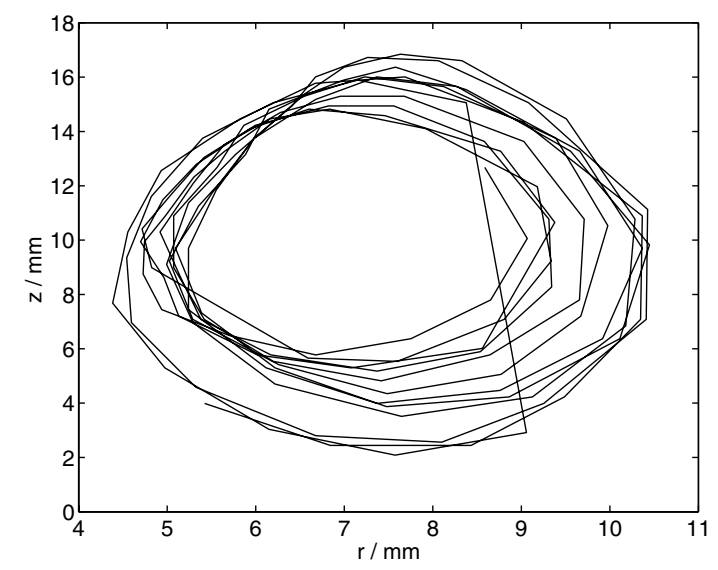

FIG. 2. Experimental results: $r(t)$ vs $z(t), Q \approx 10^{-5} \mathrm{~m}^{3} / \mathrm{s}$, $P_{a} \approx 4 \times 10^{3} \mathrm{~Pa}$, and $R_{B 0} \approx 200 \mu \mathrm{m}$.

$$
\begin{aligned}
0= & \overline{\mathbf{F}}_{A}\left(\mathbf{x}_{B}, \ddot{\mathbf{x}}_{B}\right)+\overline{\mathbf{F}}_{I}\left(\mathbf{x}_{B}\right)+\overline{\mathbf{F}}_{L}\left(\mathbf{x}_{B}, \dot{\mathbf{x}}_{B}\right) \\
& +\overline{\mathbf{F}}_{D}\left(\mathbf{x}_{B}, \dot{\mathbf{x}}_{B}\right)+\overline{\mathbf{F}}_{G}+\overline{\mathbf{F}}_{\mathrm{BJ}}\left(\mathbf{x}_{B}\right),
\end{aligned}
$$

where the overlines denote time averaging.

We will now employ models for the forces as follows: The primary Bjerknes force is

$$
\mathbf{F}_{\mathrm{BJ}}=-V_{B} \nabla P_{\text {acoustic }},
$$

in which $V_{B}$ is the bubble volume and $P_{\text {acoustic }}$ is the acoustic pressure field given by

$$
P_{\text {acoustic }}=P_{a} \frac{\sin \left(k r_{\mathrm{sp}}\right)}{k r_{\mathrm{sp}}} \sin (\omega t)
$$

$\omega$ is the angular frequency of the sound, $r_{\mathrm{sp}}=\sqrt{r^{2}+z^{2}}$ is the distance from the resonator center, $P_{a}$ is the acoustic pressure amplitude at $r_{\mathrm{sp}}=0$, and $k=\pi / R_{\text {res }}$, with $R_{\text {res }}$ the radius of the resonator. In view of the small amplitude of the driving pressure, it is safe to assume that the bubble undergoes harmonic oscillations. In this case, $V_{B}$ may be written as [4]

$$
V_{B}=V_{B 0}\left(1-3 a \frac{\sin \left(k r_{\mathrm{sp}}\right)}{k r_{\mathrm{sp}}} \sin (\omega t-\beta)\right) .
$$

$a$ is the amplitude of the oscillations of the bubble radius normalized by the undisturbed radius $R_{B 0}, V_{B 0}$ is the undisturbed bubble volume, and $\beta$ is the phase lag between the volume oscillations of the bubble and the oscillations of the sound field. The following expressions for $a$ and $\beta$ can be found from the linearized Rayleigh-Plesset equation [5]:

$$
\begin{gathered}
a=\frac{P_{a}}{R_{B 0}^{2} \rho_{l}} \frac{1}{\sqrt{\left(\omega_{0}^{2}-\omega^{2}\right)^{2}+\left(\frac{3 P_{0}}{c_{l} \rho_{l} R_{B 0}} \omega\right)^{2}}}, \\
\beta=\arctan \left(\frac{3 P_{0}}{c_{l} \rho_{l} R_{B 0}} \frac{\omega}{\omega_{0}^{2}-\omega^{2}}\right),
\end{gathered}
$$

in which $P_{0}$ is the ambient pressure, $\omega_{0}$ is the eigenfrequency of the bubble, $\rho_{l}$ is the water density, and $c_{l}$ is the speed of sound in water.

Substitution of Eqs. (4) and (5) into Eq. (3) and averaging over one acoustic cycle results in
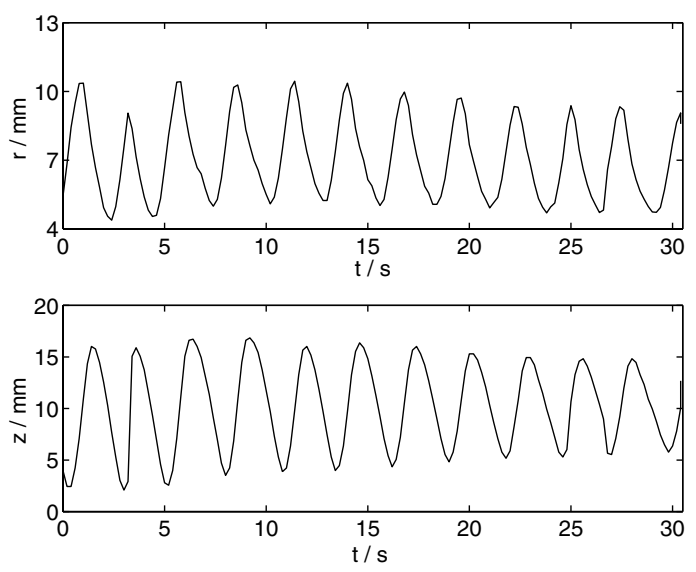

FIG. 3. Experimental results: $r(t)$ and $z(t)$ for $Q \approx$ $10^{-5} \mathrm{~m}^{3} / \mathrm{s}, P_{a} \approx 4 \times 10^{3} \mathrm{~Pa}$, and $R_{B 0} \approx 200 \mu \mathrm{m}$. From a Fourier transformation we obtain $f=0.37 \mathrm{~Hz}$. 


$$
\begin{aligned}
\overline{\mathbf{F}}_{\mathrm{BJ}}= & \frac{3}{2 r_{\mathrm{sp}}} a P_{a} V_{B 0} \frac{\sin \left(k r_{\mathrm{sp}}\right)}{k r_{\mathrm{sp}}} \cos \beta \\
& \times\left[-\frac{\sin \left(k r_{\mathrm{sp}}\right)}{k r_{\mathrm{sp}}}+\cos \left(k r_{\mathrm{sp}}\right)\right] \mathbf{e}_{r_{\mathrm{sp}}} .
\end{aligned}
$$

Since the present conditions are far from resonance, the phase lag $\beta$ is almost $\pi$ : the bubble oscillates in antiphase with the sound field, and the Bjerknes force is directed away from the center of the resonator.

Expressions for the added mass force and the inertial force acting on an oscillating bubble in a flow with small unsteadiness can be found in [6]. Averaging these expressions over one acoustic cycle results in

$$
\begin{gathered}
\overline{\mathbf{F}}_{A}=\rho_{l} C_{M} V_{B 0}\left(\frac{D \mathbf{U}}{D t}-\ddot{\mathbf{x}}_{B}\right), \\
\overline{\mathbf{F}}_{I}=\rho_{l} V_{B 0} \frac{D \mathbf{U}}{D t},
\end{gathered}
$$

where $C_{M}$ is the added-mass coefficient, which is $1 / 2$ for a sphere, whatever the Reynolds number [7]. The use of this result here is justified as $\left|\partial\left(U-U_{B}\right) / \partial t\right| \ll(U-$ $\left.U_{B}\right)^{2} / R_{B 0}$. Note that here and hereafter we assume that the average of products is the product of averages, which is justified by the large difference between the acoustic and flow time scales.

Averaging the lift force [8] on a bubble in a shear flow over one acoustic cycle gives

$$
\overline{\mathbf{F}}_{L}=C_{L} \rho_{l} V_{B 0}\left(\mathbf{U}-\dot{\mathbf{x}}_{B}\right) \times(\nabla \times \mathbf{U}),
$$

in which $C_{L}$ is the lift coefficient, which is $1 / 2$ in an inviscid weak shear flow [9]. This value is still a reasonable one down to a Reynolds number of 5 [10] and is used in the present study.

The averaged drag force is

$$
\overline{\mathbf{F}}_{D}=\frac{1}{2} \rho_{l} C_{D} \pi R_{B 0}^{2}\left|\mathbf{U}-\dot{\mathbf{x}}_{B}\right|\left(\mathbf{U}-\dot{\mathbf{x}}_{B}\right),
$$

in which $C_{D}$ is the drag coefficient, which we assume in the form $C_{D}=\frac{16}{\operatorname{Re}}\left\{1+\left[\frac{8}{\operatorname{Re}}+\frac{1}{2}\left(1+3.315 \operatorname{Re}^{-1 / 2}\right)\right]^{-1}\right\}[8]$; the Reynolds number is $\operatorname{Re}=2 R_{B 0}\left|\mathbf{U}-\mathbf{U}_{B}\right| / \nu$. Finally, the buoyancy force is

$$
\mathbf{F}_{G}=-\rho_{l} V_{B 0} \mathbf{g} .
$$

From Eqs. (6)-(11) it is immediately clear that there is no force in the $\theta$ direction, and that therefore a bubble stays in a plane with fixed $\theta$, as observed in the experiments. Figure 4 depicts the direction of the most important competing forces when the bubble has a constant downward velocity.

The numerical solution of Eq. (2) for typical parameters, $Q=2.75 \times 10^{-5} \mathrm{~m}^{3} / \mathrm{s}, \quad R_{B 0}=200 \mu \mathrm{m}$, $P_{a}=4 \times 10^{3} \mathrm{~Pa}$, and $f_{d}=20 \mathrm{kHz}$ can be seen in Fig. 5. The spiraling frequency $f$ is $0.38 \mathrm{~Hz}$ and the damping constant $\gamma$ is $0.03 \mathrm{~s}^{-1}$. These figures and Figs. 2 and 3 show that the experimental and numerical results qualitatively agree. The order of magnitude of

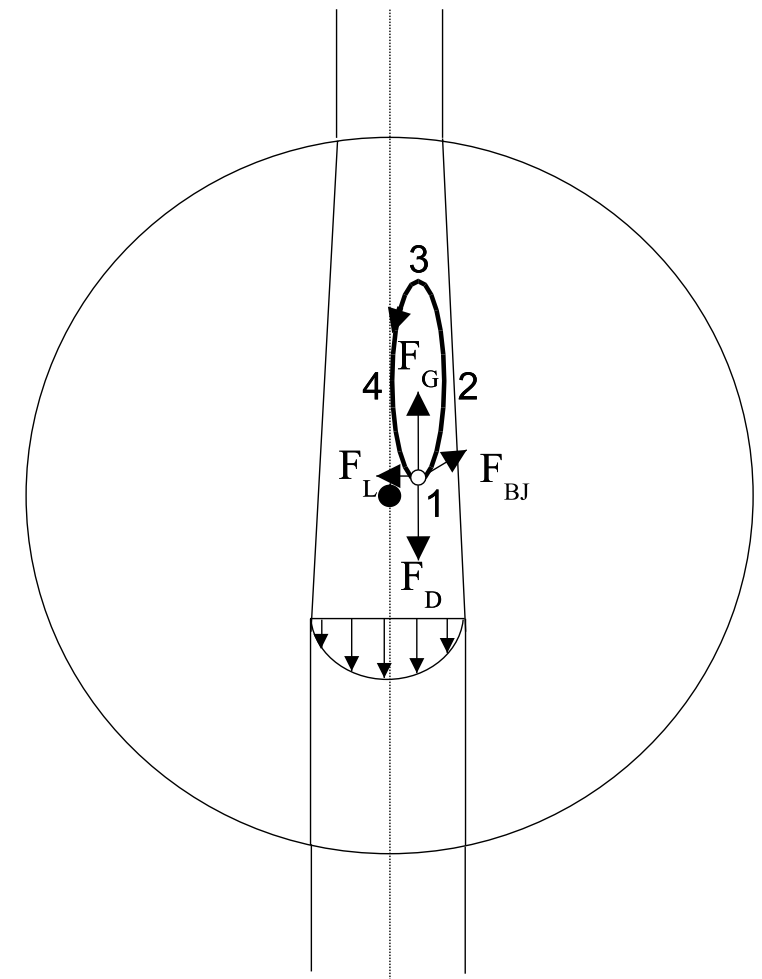

FIG. 4. Forces on the spiraling bubble when it is moving outward. During the four phases 1-4 different forces dominate, as described in the text.

the spiraling frequency and of the damping constant in the experiment and the model are the same; at present, a more quantitative comparison is not possible due to experimental inaccuracies. The physics of the experiments is, however, well reflected in the model; cf. Fig. 4, where we divided the bubble's trajectory into four phases, distinguished by the most relevant force in each one: In phase 1 the Bjerknes force pushes the bubble towards the wall. Closer to the wall, the drag force diminishes due to the reduced velocity and buoyancy takes over, letting the

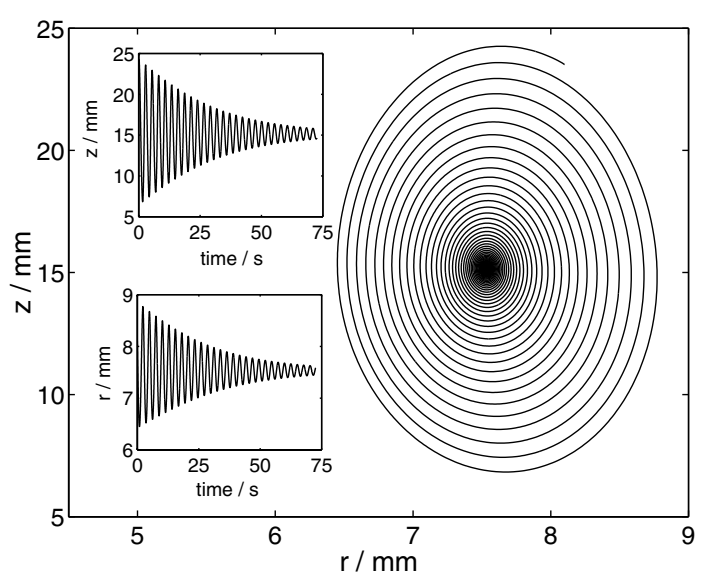

FIG. 5. Numerical results: $r(t)$ vs $z(t)$ for $Q=2.75 \times$ $10^{-5} \mathrm{~m}^{3} / \mathrm{s}, \quad P_{a}=4 \times 10^{3} \mathrm{~Pa}$, and $R_{B 0}=200 \mu \mathrm{m}$. The resulting frequency is $f=0.38 \mathrm{~Hz}$, and the resulting damping constant is $\gamma=0.03 \mathrm{~s}^{-1}$ 
bubble rise (phase 2). In this region close to the wall the lift force is larger (pointing back to the centerline), due to the increased shear. Consequently, when the bubble is sufficiently high above the center, the Bjerknes force becomes less important than the lift force which then drives the bubble back towards the centerline (phase 3 ). Here the drag can again take over, dragging the bubble downwards (phase 4) towards the region of larger Bjerknes force which eventually again takes over (phase 1). In the center of the nearly elliptical trajectory of the bubble all forces balance and the bubble is in force equilibrium.

Parameter dependence and stability analysis. - To explore the parameter space, we carried out a linear stability analysis, the results of which can be seen in Fig. 6 . The figure depicts the frequency and the damping constant of the spiraling found by the linear stability analysis and by the numerical solution of the full Eq. (2). Both results are very similar. The frequency and the damping constant are plotted as functions of the flow rate for two different driving pressures. In the lower graph one can see the damping constant $\gamma$, which is seen to be a decreasing function of the flow rate $Q$. For large enough $Q$, of course, $\gamma$ becomes negative and the bubble is swept away.

Conclusions. - We have experimentally shown the influence of acoustic forces on a bubble immersed in a shear flow. The experiments have demonstrated that the competition between hydrodynamic and acoustic forces can result, depending on the experimental parameters, in a spiraling motion. Our model of the bubble dynamics in terms of a simple force balance qualitatively reproduces the behavior observed in the experiment; a more quantitative comparison is not yet possible due to insufficient experimental accuracy. We conclude that the simple force balance (2) can be meaningfully used also in the case of combined fluid dynamical and acoustic forces approximated by timeaveraged expressions through a separation of time scales. A linear stability analysis shows that the spiraling frequency increases up to a certain flow rate and that the damping decreases. Future work must aim at improving the experimental accuracy to allow for a quantitative comparison so that eventually the lift or the added mass coefficients may be measured through this technique.

We thank G.-W. Bruggert and H. Scholten for constructing the experimental setup and L.v. Wijngaarden for dis-
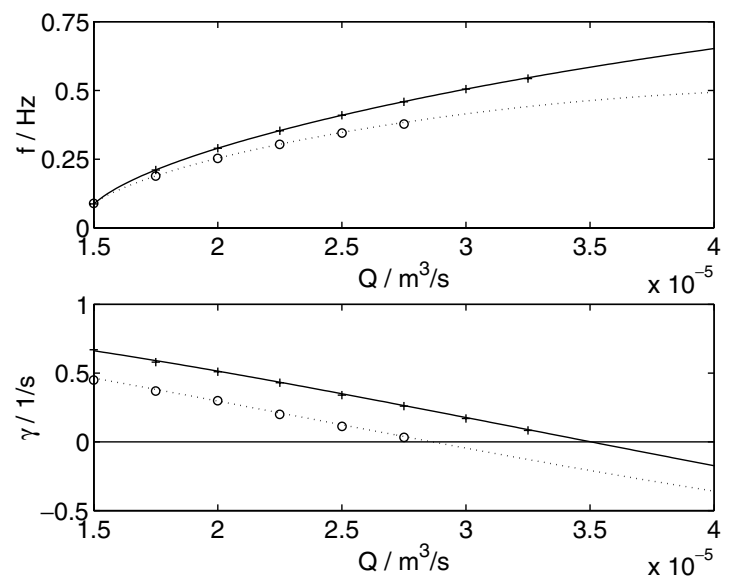

FIG. 6. Results of linear stability analysis; $f$ and $\gamma$ as functions of $Q$; lines are results of linear stability analysis, markers of the ODE model; dotted line and circles correspond to $P_{a}=4000 \mathrm{~Pa}$, solid line and crosses to $P_{a}=5000 \mathrm{~Pa}$.

cussions. The work is part of the research program of FOM, which is financially supported by NWO; A. P. acknowledges partial support from NSF.

*Corresponding author.

Email address: lohse@tn.utwente.nl

[1] K. Tachibana et al., Lancet 353, 1409 (1999).

[2] L. A. Crum, Phys. Today 47, No. 9, 22 (1994).

[3] To be more precise, over five cycles the angle $\theta$ varies by less than $5^{\circ}$. The origin of this variation presumably is a small asymmetry of the cell.

[4] T. G. Leighton, The Acoustic Bubble (Academic Press, London, 1996).

[5] C. E. Brennen, Cavitation and Bubble Dynamics (Oxford University Press, Oxford, 1995).

[6] J. Magnaudet and D. Legendre, Phys. Fluids 10, 550 (1998).

[7] M. Rivero, J. Magnaudet, and J. Fabre, C.R. Acad. Sci. Paris Série II 312, 1499 (1991).

[8] J. Magnaudet and I. Eames, Annu. Rev. Fluid Mech. 32, 659 (2000).

[9] T. R. Auton, J. Fluid Mech. 183, 199 (1987).

[10] D. Legendre and J. Magnaudet, J. Fluid Mech. 368, 81 (1998). 